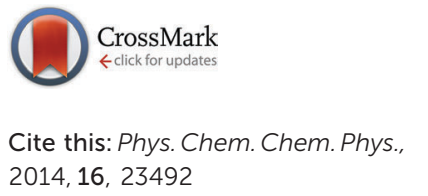

DOI: $10.1039 /$ c4cp90145b

www.rsc.org/pccp

\section{Correction: Asymmetric bi-layer PFSA membranes as model systems for the study of water management in the PEMFC}

\author{
A. Z. Peng, ${ }^{\text {ab }}$ A. Morin, ${ }^{\star a}$ P. Huguet, ${ }^{b}$ Y. Lanteri ${ }^{b}$ and S. Deabate ${ }^{\star b}$
}

Correction for 'Asymmetric bi-layer PFSA membranes as model systems for the study of water management in the PEMFC' by A. Z. Peng et al., Phys. Chem. Chem. Phys., 2014, 16, 20941-20956.

The name of the first listed author of the article has been incorrectly displayed as A. Z. Peng. The correct name is Z. Peng. The Royal Society of Chemistry apologises for these errors and any consequent inconvenience to authors and readers.

\footnotetext{
${ }^{a}$ LITEN-DEHT-LCPEM, CEA-Grenoble, 17 rue des Martyrs, 38054 Grenoble cedex 9, France. E-mail: arnaud.morin@cea.fr; Tel: +33-4-38-78-59-86

${ }^{b}$ IEM (Institut Européen des Membranes) UMR 5635 (CNRS-ENSCM-UM2) Université Montpellier 2, Place E. Bataillon, F-34095, Montpellier, France.

E-mail: stefano.deabate@univ-montp2.fr; Fax: +33-4-67-14-91-19; Tel: +33-4-67-14-91-12
} 